\title{
1 \\ Three problems \\ for the clairvoyant demon
}

Geoffrey Grimmett

\begin{abstract}
A number of tricky problems in probability are discussed, having in common one or more infinite sequences of coin tosses, and a representation as a problem in dependent percolation. Three of these problems are of 'Winkler' type, that is, they are challenges for a clairvoyant demon.
\end{abstract}


Edited by 


\subsection{Introduction}

Probability theory has emerged in recent decades as a crossroads where many sub-disciplines of mathematical science meet and interact. Of the many examples within mathematics, we mention (not in order): analysis, partial differential equations, mathematical physics, measure theory, discrete mathematics, theoretical computer science, and number theory. The International Mathematical Union and the Abel Memorial Fund have recently accorded acclaim to probabilists. This process of recognition by others has been too slow, and would have been slower without the efforts of distinguished mathematicians including John Kingman.

JFCK's work looks towards both theory and applications. To single out just two of his theorems: the subadditive ergodic theorem $[21,22]$ is a piece of mathematical perfection which has also proved rather useful in practice; his 'coalescent' $[23,24]$ is a beautiful piece of probability, now a keystone of mathematical genetics. John is also an inspiring and devoted lecturer, who continued to lecture to undergraduates even as the Bristol Vice-Chancellor, and the Director of the Isaac Newton Institute in Cambridge. Indeed, the current author learned his measure and probability from partial attendance at John's course in Oxford in 1970/71.

To misquote Frank Spitzer [34, Sect. 8], we turn to a very down-toearth problem: consider an infinite sequence of light bulbs. The basic commodity of probability is an infinite sequence of coin tosses. Such a sequence has been studied for so long, and yet there remain 'simple to state' problems that appear very hard. We present some of these problems here. Sections 1.3-1.5 are devoted to three famous problems for the so-called clairvoyant demon, a presumably non-human being to whom is revealed the (infinite) realization of the sequence, and who is permitted to plan accordingly for the future.

Each of these problems may be phrased as a geometrical problem of percolation type. The difference with classical percolation [13] lies in the dependence of the site variables. Percolation is reviewed briefly in Section 1.2. This article ends with two short sections on related problems, namely: other forms of dependent percolation, and the question of 'percolation of words'. 


\subsection{Site percolation}

We set the scene by reminding the reader of the classical 'site percolation model' of Broadbent and Hammersley [9]. Consider a countably infinite, connected graph $G=(V, E)$. To each 'site' $v \in V$ we assign a Bernoulli random variable $\omega(v)$ with density $p$. That is, $\omega=\{\omega(v): v \in V\}$ is a family of independent, identically distributed random variables taking the values 0 and 1 with respective probabilities $1-p$ and $p$. A vertex $v$ is called open if $\omega(v)=1$, and closed otherwise.

Let 0 be a given vertex, called the origin, and let $\theta(p)$ be the probability that the origin lies in an infinite open self-avoiding path of $G$. It is clear that $\theta$ is non-decreasing in $p$, and $\theta(0)=0, \theta(1)=1$. The critical probability is given as

$$
p_{\mathrm{c}}=p_{\mathrm{c}}(G):=\sup \{p: \theta(p)=0\} .
$$

It is a standard exercise to show that the value of $p_{\mathrm{c}}$ does not depend on the choice of origin, but only on the graph $G$.

One may instead associate the random variables with the edges of the graph, rather than the vertices, in which case the process is termed 'bond percolation'. Percolation is recognised as a fundamental model for a random medium. It is important in probability and statistical physics, and it continues to be the source of beautiful and apparently hard mathematical problems, of which the most outstanding is to prove that $\theta\left(p_{\mathrm{c}}\right)=0$ for the three-dimensional lattice $\mathbb{Z}^{3}$. Of the several recent accounts of the percolation model, we mention $[13,14]$.

Most attention has been paid to the case when $G$ is a crystalline lattice in two or more dimensions. The current article is entirely concerned with aspects of two-dimensional percolation, particularly on the square and triangular lattices illustrated in Figure 1.1. Site percolation on the triangular lattice has featured prominently in the news in recent years, owing to the work of Smirnov, Lawler-Schramm-Werner, and others on the relationship of this model (with $p=p_{\mathrm{c}}=\frac{1}{2}$ ) to the process of random curves in $\mathbb{R}^{2}$ termed Schramm-Löwner evolutions (SLE), and particularly the process denoted $\mathrm{SLE}_{6}$. See [35].

When $G$ is a directed graph, one may ask about the existence of an infinite open directed path from the origin, in which case the process is referred to as directed (or oriented) percolation.

Variants of the percolation model are discussed in the following sections, with the emphasis on models with site/bond variables that are dependent. 

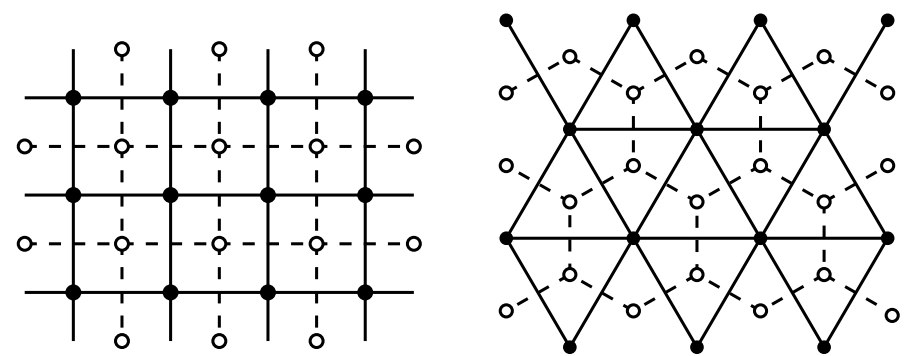

Figure 1.1 The square lattice $\mathbb{Z}^{2}$ and the triangular lattice $\mathbb{T}$, with their dual lattices.

\subsection{Clairvoyant scheduling}

Let $G=(V, E)$ be a finite connected graph. A symmetric random walk on $G$ is a Markov chain $X=\left(X_{k}: k=0,1,2, \ldots\right)$ on the state space $V$, with transition matrix

$$
\mathbb{P}\left(X_{k+1}=w \mid X_{k}=v\right)= \begin{cases}\frac{1}{\Delta_{v}} & \text { if } v \sim w, \\ 0 & \text { if } v \nsim w\end{cases}
$$

where $\Delta_{v}$ is the degree of vertex $v$, and $\sim$ denotes the adjacency relation of $G$. Random walks on general graphs have attracted much interest in recent years, see [14, Chap. 1] for example.

Let $X$ and $Y$ be independent random walks on $G$ with distinct starting sites $x_{0}, y_{0}$, respectively. We think of $X$ (respectively, $Y$ ) as describing the trajectory of a particle labelled $X$ (respectively, $Y$ ) around $G$. A clairvoyant demon is set the task of keeping the walks apart from one another for all time. To this end, (s)he is permitted to schedule the walks in such a way that exactly one walker moves at each epoch of time. Thus, the walks may be delayed, but they are required to follow their prescribed trajectories.

More precisely, a schedule is defined as a sequence $Z=\left(Z_{1}, Z_{2}, \ldots\right)$ in the space $\{X, Y\}^{\mathbb{N}}$, and a given schedule $Z$ is implemented in the following way. From the $X$ and $Y$ trajectories, we construct the rescheduled walks $Z(X)$ and $Z(Y)$, where:

1. If $Z_{1}=X$, the $X$-particle takes one step at time 1 , and the $Y$-particle remains stationary. If $Z_{1}=Y$, it is the $Y$-particle that moves, and 
the $X$-particle that remains stationary. Thus,

$$
\begin{array}{lll}
\text { if } Z_{1}=X & \text { then } \quad Z(X)_{1}=X_{1}, Z(Y)_{1}=Y_{0}, \\
\text { if } Z_{1}=Y & \text { then } \quad Z(X)_{1}=X_{0}, Z(Y)_{1}=Y_{1} .
\end{array}
$$

2. Assume that, after time $k$, the $X$-particle has made $r$ moves and the $Y$-particle $k-r$ moves, so that $Z(X)_{k}=X_{r}$ and $Z(Y)_{k}=Y_{k-r}$.

$$
\begin{array}{ll}
\text { If } Z_{k+1}=X & \text { then } \quad Z(X)_{k+1}=X_{r+1}, Z(Y)_{k+1}=Y_{k-r}, \\
\text { if } Z_{k+1}=Y & \text { then } \quad Z(X)_{k+1}=X_{r}, \quad Z(Y)_{k+1}=Y_{k-r+1} .
\end{array}
$$

We call the schedule $Z \operatorname{good}$ if $Z(X)_{k} \neq Z(Y)_{k}$ for all $k \geq 1$, and we say that the demon succeeds if there exists a good schedule $Z=Z(X, Y)$. (We overlook issues of measurability here.) The probability of success is

$$
\theta(G):=\mathbb{P}(\text { there exists a good schedule), }
$$

and we ask: for which graphs $G$ is it the case that $\theta(G)>0$ ? This question was posed by Peter Winkler (see the discussion in $[10,11]$ ). Note that the answer is independent of the choice of (distinct) starting points $x_{0}, y_{0}$.

The problem takes a simpler form when $G$ is the complete graph on some number, $M$ say, of vertices. In order to simplify it still further, we add a loop to each vertex. Write $V=\{1,2, \ldots, M\}$, and $\theta(M):=$ $\theta(G)$. A random walk on $G$ is now a sequence of independent, identically distributed points in $\{1,2, \ldots, M\}$, each with the uniform distribution. It is expected that $\theta(M)$ is non-decreasing in $M$, and it is clear by coupling that $\theta(k M) \geq \theta(M)$ for $k \geq 1$. Also, it is not too hard to show that $\theta(3)=0$.

Question 1.1 Is it the case that $\theta(M)>0$ for sufficiently large $M$ ? Perhaps $\theta(4)>0$ ?

This problem has a geometrical formulation of percolation-type. Consider the positive quadrant $\mathbb{Z}_{+}^{2}=\{(i, j): i, j=0,1,2, \ldots\}$ of the square lattice $\mathbb{Z}^{2}$. A path is taken to be an infinite sequence $\left(u_{n}, v_{n}\right), n \geq 0$, with $\left(u_{0}, v_{0}\right)=(0,0)$ such that, for all $n \geq 0$,

either $\left(u_{n+1}, v_{n+1}\right)=\left(u_{n}+1, v_{n}\right) \quad$ or $\quad\left(u_{n+1}, v_{n+1}\right)=\left(u_{n}, v_{n}+1\right)$.

With $X, Y$ the random walks as above, we declare the vertex $(i, j)$ to be open if $X_{i} \neq Y_{j}$. It may be seen that the demon succeeds if and only if there exists a path all of whose vertices are open.

Some discussion of this problem may be found in [11]. The law of the 
open vertices is 3 -wise independent but not 4 -wise independent, in the sense of language introduced in Section 1.6.

The problem becomes significantly easier if paths are allowed to be undirected. For the totally undirected problem, it is proved in $[3,36]$ that there exists an infinite open path with strictly positive probability if and only if $M \geq 4$.

\subsection{Clairvoyant compatibility}

Let $p \in(0,1)$, and let $X_{1}, X_{2}, \ldots$ and $Y_{1}, Y_{2}, \ldots$ be independent sequences of independent Bernoulli variables with common parameter $p$. We say that a collision occurs at time $n$ if $X_{n}=Y_{n}=1$. The demon is now charged with the removal of collisions, and to this end (s)he is permitted to remove 0 s from the sequences.

Let $\mathbb{N}=\{1,2, \ldots\}$ and $\mathcal{W}=\{0,1\}^{\mathbb{N}}$, the set of singly-infinite sequences of 0 s and $1 \mathrm{~s}$. Each $w \in \mathcal{W}$ is considered as a word in an alphabet of two letters, and we generally write $w_{n}$ for its $n$th letter. For $w \in \mathcal{W}$, there exists a sequence $i(w)=\left(i(w)_{1}, i(w)_{2}, \ldots\right)$ of non-negative integers such that $w=0^{i_{1}} 10^{i_{2}} 1 \cdots$, that is, there are exactly $i_{j}=i(w)_{j}$ zeros between the $(j-1)$ th and $j$ th appearances of 1 . For $x, y \in \mathcal{W}$, we write $x \rightarrow y$ if $i(x)_{j} \geq i(y)_{j}$ for $j \geq 1$. That is, $x \rightarrow y$ if and only if $y$ may be obtained from $x$ by the removal of 0 s.

Two infinite words $v, w$ are said to be compatible if there exist $v^{\prime}$ and $w^{\prime}$ such that $v \rightarrow v^{\prime}, w \rightarrow w^{\prime}$, and $v_{n}^{\prime} w_{n}^{\prime}=0$ for all $n$. For given realizations $X, Y$, we say that the demon succeeds if $X$ and $Y$ are compatible. Write

$$
\psi(p)=\mathbb{P}_{p}(X \text { and } Y \text { are compatible }) .
$$

Note that, by a coupling argument, $\psi$ is a non-increasing function.

Question 1.2 For what $p$ is it the case that $\psi(p)>0$.

It is easy to see as follows that $\psi\left(\frac{1}{2}\right)=0$. When $p=\frac{1}{2}$, there exists almost surely an integer $N$ such that

$$
\sum_{i=1}^{N} X_{i}>\frac{1}{2} N, \quad \sum_{i=1}^{N} Y_{i}>\frac{1}{2} N .
$$

With $N$ chosen thus, it is not possible for the demon to prevent a collision in the first $N$ values. By working more carefully, one may obtain that $\psi\left(\frac{1}{2}-\epsilon\right)=0$ for small positive $\epsilon$, see the discussion in [12]. 
Gács has proved in [12] that $\psi\left(10^{-400}\right)>0$, and he has noted that there is room for improvement.

\subsection{Clairvoyant embedding}

The clairvoyant demon's third problem stems from work on long-range percolation of words (see Section 1.7). Let $X_{1}, X_{2}, \ldots$ and $Y_{1}, Y_{2}, \ldots$ be independent sequences of independent Bernoulli variables with parameter $\frac{1}{2}$. Let $M \in\{2,3, \ldots\}$. The demon's task is to find a monotonic embedding of the $X_{i}$ within the $Y_{j}$ in such a way that the gaps between successive terms are no greater than $M$.

Let $v, w \in \mathcal{W}$. We say that $v$ is $M$-embeddable in $w$, and we write $v \subseteq_{M} w$, if there exists an increasing sequence $\left(m_{i}: i \geq 1\right)$ of positive integers such that $v_{i}=w_{m_{i}}$ and $1 \leq m_{i}-m_{i-1} \leq M$ for all $i \geq 1$. (We set $m_{0}=0$.) A similar definition is made for finite words $v$ lying in one of the spaces $\mathcal{W}_{n}=\{0,1\}^{n}, n \geq 1$.

The demon succeeds in the above task if $X \subseteq_{M} Y$, and we let

$$
\rho(M)=\mathbb{P}\left(X \subseteq_{M} Y\right)
$$

It is elementary that $\rho(M)$ is non-decreasing in $M$.

Question 1.3 Is it the case that $\rho(M)>0$ for sufficiently large $M$ ?

This question is introduced and discussed in [15], and partial but limited results proved. One approach is to estimate the first two moments of the number $N_{n}(w)$ of $M$-embeddings of the finite word $w=$ $w_{1} w_{2} \cdots w_{n} \in \mathcal{W}_{n}$ within the random word $Y$. It is elementary that $E\left(N_{n}(w)\right)=(M / 2)^{n}$ for any such $w$, and it may be shown that

$$
\frac{E\left(N_{n}(X)^{2}\right)}{E\left(N_{n}(X)\right)^{2}} \sim A_{M} c_{M}^{n} \quad \text { as } n \rightarrow \infty,
$$

where $A_{M}>0$ and $c_{M}>1$ for $M \geq 2$. The fact that $E\left(N_{n}(w)\right) \equiv 1$ when $M=2$ is strongly suggestive that $\rho(2)=0$, and this is part of the next theorem.

Theorem 1.4 [15] We have that $\rho(2)=0$. Furthermore, for $M=2$,

$$
\mathbb{P}\left(w \subseteq_{2} Y\right) \leq \mathbb{P}\left(a_{n} \subseteq_{2} Y\right) \quad \text { for all } w \in \mathcal{W}_{n}
$$

where $a_{n}=0101 \cdots$ is the alternating word of length $n$. 
It is immediate that (1.5) implies $\rho(2)=0$ on noting that, for any infinite periodic word $\pi, \mathbb{P}\left(\pi \subseteq_{M} Y\right)=0$ for all $M \geq 2$. One may estimate such probabilities more exactly through solving appropriate difference equations. For example, $v_{n}(M)=\mathbb{P}\left(a_{n} \subseteq_{M} Y\right)$ satisfies

$$
v_{n+1}(M)=(\alpha+(M-1) \beta) v_{n}-\beta(M-2 \alpha) v_{n-1}, \quad n \geq 1,
$$

with boundary conditions $v_{0}(M)=1, v_{1}(M)=\alpha$. Here,

$$
\alpha+\beta=1, \quad \beta=2^{-M} .
$$

The characteristic polynomial associated with (1.6) is a quadratic with one root in each of the disjoint intervals $(0, M \beta)$ and $(\alpha, 1)$. The larger root equals $1-(1+\mathrm{o}(1)) 2^{1-2 M}$ for large $M$, so that, in rough terms

$$
v_{n}(M) \approx\left(1-2^{1-2 M}\right)^{n} .
$$

Herein lies a health warning for simulators. One knows that, almost surely, $a_{n} \nsubseteq_{M} Y$ for large $n$, but one has to look on scales of order $2^{2 M-1}$ if one is to observe its extinction with reasonable probability.

One may ask about the 'best' and 'worst' words. Inequality (1.5) asserts that an alternating word $a_{n}$ is the most easily embedded word when $M=2$. It is not known which word is best when $M>2$. Were this a periodic word, it would follow that $\rho(M)=0$. Unsurprisingly, the worst word is a constant word $c_{n}$ (of which there are of course two). That is, for all $M \geq 2$,

$$
\mathbb{P}\left(w \subseteq_{M} Y\right) \geq \mathbb{P}\left(c_{n} \subseteq_{M} Y\right) \quad \text { for all } w \in \mathcal{W}_{n},
$$

where, for definiteness, we set $c_{n}=1^{n} \in \mathcal{W}_{n}$.

Let $M=2$, so that the mean number $E\left(N_{n}(w)\right)$ of embeddings of any word of length $n$ is exactly 1 (as remarked above). A further argument is required to deduce that $\rho(2)=0$. Peled [32] has made rigorous the following alternative to that used in the proof of Theorem 1.4. Assume that the word $w \in \mathcal{W}_{n}$ satisfies $w \subseteq_{2} Y$. For some small $c>0$, one may identify (for most embeddings, with high probability) $\mathrm{cn}$ positions at which the embedding may be altered, independently of each other. This gives $2^{c n}$ possible 'local variations' of the embedding. It may be deduced that the probability of embedding a word $w \in \mathcal{W}_{n}$ is exponentially small in $n$, and also $\rho(2)=0$.

The sequences $X, Y$ have been taken above with parameter $\frac{1}{2}$. Little changes with Question 1.3 in a more general setting. Let the two (respective) parameters be $p_{X}, p_{Y} \in(0,1)$. It turns out that the validity of 
the statement "for all $M \geq 2, \mathbb{P}\left(X \subseteq_{M} Y\right)=0$ " is independent of the values of $p_{X}, p_{Y}$. On the other hand, (1.5) is not generally true. See [15].

A number of easier variations on Question 1.3 spring immediately to mind, of which two are mentioned here.

1. Suppose the gap between the embeddings of $X_{i-1}$ and $X_{i}$ must be bounded above by some $M_{i}$. How slow a growth on the $M_{i}$ suffices that the embedding probability be strictly positive? [An elementary bound follows by the Borel-Cantelli lemma.]

2. Suppose that the demon is allowed to look only boundedly into the future. How much clairvoyance may (s)he be allowed without the embedding probability becoming strictly positive?

Further questions (and variations thereof) have been proposed by others.

1. In a 'penalised embedding' problem, we are permitted mismatches by paying a (multiplicative) penalty $b$ for each. What is the cost of the 'cheapest' penalised embedding of the first $n$ terms, and what can be said as $b \rightarrow \infty$ ? [Erwin Bolthausen]

2. What can be said if we are required to embed only the $1 \mathrm{~s}$ ? That is, a ' 1 ' must be matched to a ' 1 ', but a ' 0 ' may be matched to either ' 0 ' or ' 1 '. [Simon Griffiths]

3. The above problems may be described as embedding $\mathbb{Z}$ in $\mathbb{Z}$. In this language, might it be possible to embed $\mathbb{Z}^{m}$ in $\mathbb{Z}^{n}$ for some $m, n \geq 2$ ? [Ron Peled]

Question 1.3 may be expressed as a geometrical problem of percolation type. With $X$ and $Y$ as above, we declare the vertex $(i, j) \in \mathbb{N}^{2}$ to be open if $X_{i}=Y_{j}$. A path is defined as an infinite sequence $\left(u_{n}, v_{n}\right), n \geq 0$, of vertices such that:

$$
\left(u_{0}, v_{0}\right)=(0,0), \quad\left(u_{n+1}, v_{n+1}\right)=\left(u_{n}+1, v_{n}+d_{n}\right),
$$

for some $d_{n}$ satisfying $1 \leq d_{n} \leq M$. It is easily seen that $X \subseteq_{M} Y$ if and only if there exists a path all of whose vertices are open. (We declare $(0,0)$ to be open.)

With this formulation in mind, the above problem may be represented by the icon at the top left of Figure 1.2. The further icons of that figure represent examples of problems of similar type. Nothing seems to be known about these except that:

1. the argument of Peled [32] may be applied to problem (b) with $M=2$ to obtain that $\mathbb{P}\left(w \subseteq_{2} Y\right)=0$ for all $w \in \mathcal{W}$, 


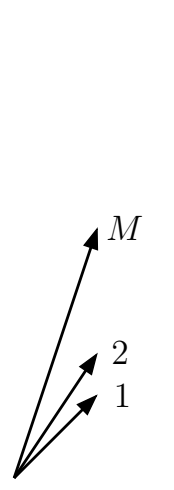

(a)

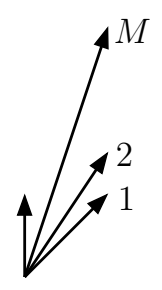

(d)

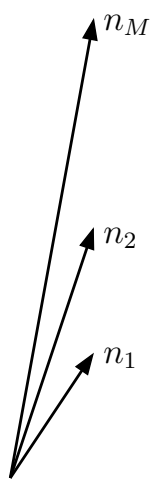

(b)

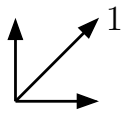

(e)

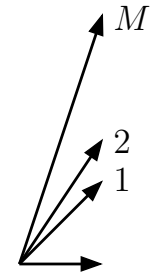

(c)

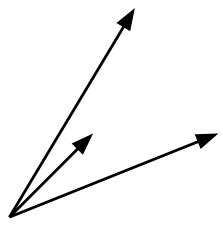

(f)

Figure 1.2 Icons describing a variety of embedding problems.

2. problem (e) is easily seen to be trivial.

It is, as one might expect, much easier to embed words in two dimensions than in one, and indeed this may be done along a path of $\mathbb{Z}^{2}$ that is directed in the north-easterly direction. This statement is made more precise as follows. Let $Y=\left(Y_{i, j}: i, j=1,2, \ldots\right)$ be a two-dimensional array of independent Bernoulli variables with parameter $p \in(0,1)$, say. A word $v \in \mathcal{W}$ is said to be $M$-embeddable in $Y$, written $v \subseteq_{M} Y$, if there exist strictly increasing sequences $\left(m_{i}: i \geq 1\right),\left(n_{i}: i \geq 1\right)$ of positive integers such that $v_{i}=Y_{m_{i}, n_{i}}$ and

$$
1 \leq\left(m_{i}-m_{i-1}\right)+\left(n_{i}-n_{i-1}\right) \leq M, \quad i \geq 1 .
$$

(We set $m_{0}=n_{0}=0$.) The following answers a question posed in [29]. Note added at revision: A related result has been discovered independently in [30]. 
Theorem 1.7 [14] Suppose $R \geq 1$ is such that $1-p^{R^{2}}-(1-p)^{R^{2}}>\vec{p}_{\mathrm{c}}$, the critical probability of directed site percolation on $\mathbb{Z}^{2}$. With strictly positive probability, every infinite word $w$ satisfies $w \subseteq_{5 R} Y$.

The identification of the set of words that are 1-embeddable in the twodimensional array $Y$, with positive probability, is much harder. This is a problem of percolation of words, and the results to date are summarised in Section 1.7.

Proof We use a block argument. Let $R \in\{2,3, \ldots\}$. For $(i, j) \in \mathbb{N}^{2}$, define the block $B_{R}(i, j)=((i-1) R, i R] \times((j-1) R, j R] \subseteq \mathbb{N}^{2}$. On the graph of blocks, we define the (directed) relation $B_{R}(i, j) \rightarrow B_{R}(m, n)$ if $(m, n)$ is either $(i+1, j+1)$ or $(i+1, j+2)$. By drawing a picture, one sees that the ensuing directed graph is isomorphic to $\mathbb{N}^{2}$ directed north-easterly. Note that the $L^{1}$-distance between two vertices lying in adjacent blocks is no more than $5 R$.

We call a block $B_{R}$ good if it contains at least one 0 and at least one 1. It is trivial that

$$
\mathbb{P}_{p}\left(B_{R} \text { is good }\right)=1-p^{R^{2}}-(1-p)^{R^{2}} .
$$

If the right side exceeds the critical probability $\vec{p}_{\mathrm{c}}$ of directed site percolation on $\mathbb{Z}^{2}$, then there is a strictly positive probability of an infinite directed path of good blocks in the block graph, beginning at $B_{R}(1,1)$. Such a path contains $5 R$-embeddings of all words.

The problem of clairvoyant embedding is connected to a question concerning isometries of random metric spaces discussed in [33]. In broad terms, two metric spaces $\left(S_{i}, \mu_{i}\right), i=1,2$, are said to be 'quasi-isometric' (or 'roughly isometric') if their metric structure is the same up to multiplicative and additive constants. That is, there exists a mapping $T$ : $S_{1} \rightarrow S_{2}$ and positive constants $M, D, R$ such that:

$$
\frac{1}{M} \mu_{1}(x, y)-D \leq \mu_{2}(T(x), T(y)) \leq M \mu_{1}(x, y)+D, \quad x, y \in S_{1},
$$

and, for $x_{2} \in S_{2}$, there exists $x_{1} \in S_{1}$ with $\mu_{2}\left(x_{2}, T\left(x_{1}\right)\right) \leq R$.

It has been asked whether two Poisson process on the line, viewed as random sets with metric inherited from $\mathbb{R}$, are quasi-isometric. This question is open at the time of writing. A number of related results are proved in [33], where a history of the problem may be found also. It turns out that the above question is equivalent to the following. Let $X=$ $\left(\ldots, X_{-1}, X_{0}, X_{1}, \ldots\right)$ be a sequence of independent Bernoulli variables with common parameter $p_{X}$. The sequence $X$ generates a random metric 
space with points $\left\{i: X_{i}=1\right\}$ and metric inherited from $\mathbb{Z}$. Is it the case that two independent sequences $X$ and $Y$ generate quasi-isometric metric spaces? A possibly important difference between this problem and clairvoyant embedding is that quasi-isometries of metric subspaces of $\mathbb{Z}$ need not be monotone.

\subsection{Dependent percolation}

Whereas there is only one type of independence, there are many types of dependence, too many to be summarised here. We mention just three further types of dependent percolation in this section, of which the first (at least) arises in the context of processes in random environments. In each, the dependence has infinite range, and in this sense these problems have something in common with those treated in Sections 1.3-1.5.

For our first example, let $X=\left\{X_{i}: i \in \mathbb{Z}\right\}$ be independent, identically distributed random variables taking values in $[0,1]$. Conditional on $X$, the vertex $(i, j)$ of $\mathbb{Z}^{2}$ is declared open with probability $X_{i}$, and different vertices receive (conditionally) independent states. The ensuing measure possesses a dependence that extends without limit in the vertical direction. Let $p_{\mathrm{c}}$ denote the critical probability of site percolation on $\mathbb{Z}^{2}$. If the law $\mu$ of $X_{0}$ places probability both below and above $p_{\mathrm{c}}$, there exist (almost surely) vertically-unbounded domains that consider themselves subcritical, and others that consider themselves supercritical. Depending on the choice of $\mu$, the process may or may not possess infinite open paths, and necessary and sufficient conditions have proved elusive. The most successful technique for dealing with such problems seems to be the so-called 'multiscale analysis'. This leads to sufficient conditions under which the process is subcritical (respectively, supercritical). See [25, 26].

There is a variety of models of physics and applied probability for which the natural random environment is exactly of the above type. Consider, for example, the contact model in $d$ dimensions with recovery rates $\delta_{x}$ and infection rates $\lambda_{e}$, see $[27,28]$. Suppose that the environment is randomised through the assumption that the $\delta_{x}$ (respectively, $\lambda_{e}$ ) are independent and identically distributed. The graphical representation of this model may be viewed as a 'vertically directed' percolation model on $\mathbb{Z}^{d} \times[0, \infty)$, in which the intensities of infections and recoveries are dependent in the vertical direction. See $[1,8,31]$ for further discussion.

Vertical dependence arises naturally in certain models of statistical physics also, of which we present one example. The 'quantum Ising 
model' on a graph $G$ may be formulated as a problem in stochastic geometry on a product space of the form $G \times[0, \beta]$, where $\beta$ is the inverse temperature. A fair bit of work has been done on the quantum model in a random environment, that is, when its parameters vary randomly around different vertices/edges of $G$. The corresponding stochastic model on $G \times[0, \beta]$ has 'vertical dependence' of infinite range. See $[7,16]$.

It is easy to adapt the above structure to provide dependencies in both horizontal and vertical directions, although the ensuing problems may be considered (so far) to have greater mathematical than physical interest. For example, consider bond percolation on $\mathbb{Z}^{2}$, in which the states of horizontal edges are correlated thus, and similarly those of vertical edges. A related three-dimensional system has been studied by Jonasson, Mossel, and Peres [18]. Draw planes in $\mathbb{R}^{3}$ orthogonal to the $x$-axis, such that they intersect the $x$-axis at points of a Poisson process with given intensity $\lambda$. Similarly, draw independent families of planes orthogonal to the $y$ - and $z$-axes. These three families define a 'stretched' copy of $\mathbb{Z}^{3}$. An edge of this stretched lattice, of length $l$, is declared to be open with probability $e^{-l}$, independently of the states of other edges. It is proved in [18] that, for sufficiently large $\lambda$, there exists (a.s.) an infinite open directed percolation cluster that is transient for simple random walk. The method of proof is interesting, proceeding as it does by the method of 'exponential intersection tails' (EIT) of [5]. When combined with an earlier argument of Häggström, this proves the existence of a percolation phase transition for the model.

The method of EIT is invalid in two dimensions, because random walk is recurrent on $\mathbb{Z}^{2}$. The corresponding percolation question in two dimensions was answered using different means by Hoffman [17].

In our final example, the dependence comes without geometrical information. Let $k \geq 2$, and call a family of random variables $k$-wise independent if any $k$-subset is independent. Note that the vertex-states arising in the clairvoyant scheduling problem of Section 1.3 are 3 -wise independent but not 4-wise independent.

Benjamini, Gurel-Gurevich, and Peled [6] have investigated various properties of $k$-wise independent Bernoulli families, and in particular the following percolation question. Consider the $n$-box $B_{n}=[1, n]^{d}$ in $\mathbb{Z}^{d}$ with $d \geq 2$, in which the measure governing the site variables $\{\omega(v)$ : $\left.v \in B_{n}\right\}$ has local density $p$ and is $k$-wise independent. Let $L_{n}$ be the event that two given opposite faces are connected by an open path in the box. Thus, for large $n$, the probability of $L_{n}$ under the product measure $\mathbb{P}_{p}$ has a sharp threshold around $p=p_{\mathrm{c}}\left(\mathbb{Z}^{d}\right)$. The problem is to find 
bounds on the smallest value of $k$ such that the probability of $L_{n}$ is close to its value $\mathbb{P}_{p}\left(L_{n}\right)$ under product measure.

This question may be formalised as follows. Let $\Pi=\Pi(n, k, p)$ be the set of probability measures on $\{0,1\}^{B_{n}}$ that have density $p$ and are $k$-wise independent. Let

$$
\epsilon_{n}(p, k)=\max _{\mathbb{P} \in \Pi} \mathbb{P}\left(L_{n}\right)-\min _{\mathbb{P} \in \Pi} \mathbb{P}\left(L_{n}\right),
$$

and

$$
K_{n}(p)=\min \left\{k: \epsilon_{n}(p, k) \leq \delta\right\},
$$

where for definiteness we may take $\delta=0.01$ as in [6]. Thus, roughly speaking, $K_{n}(p)$ is a quantification of the amount of independence required in order that, for all $\mathbb{P} \in \Pi, \mathbb{P}\left(L_{n}\right)$ differs from $\mathbb{P}_{p}\left(L_{n}\right)$ by at most $\delta$.

Benjamini, Gurel-Gurevich, and Peled have proved, in an ongoing project [6], that $K_{n}(p) \leq c \log n$ when $d=2$ and $p \neq p_{c}$ (and when $d>2$ and $p<p_{\mathrm{c}}$ ), for some constant $c=c(p, d)$. They have in addition a lower bound for $K_{n}(p)$ that depends on $p, d$, and $n$, and goes to $\infty$ as $n \rightarrow \infty$.

\subsection{Percolation of words}

Recall the set $\mathcal{W}=\{0,1\}^{\mathbb{N}}$ of words in the alphabet comprising the two letters 0,1 . Consider the site percolation process of Section 1.2 on a countably infinite connected graph $G=(V, E)$, and write $\omega=$ $\{\omega(v): v \in V\}$ for the ensuing configuration. Let $v \in V$ and let $\mathcal{S}_{v}$ be the set of all self-avoiding walks starting at $v$. Each $\pi \in \mathcal{S}_{v}$ is a path $v_{0}, v_{1}, v_{2}, \ldots$ with $v_{0}=v$. With the path $\pi$ we associate the word $w(\pi)=$ $\omega\left(v_{1}\right) \omega\left(v_{2}\right) \cdots$, and we write $\mathcal{W}_{v}=\left\{w(\pi): \pi \in \mathcal{S}_{v}\right\}$ for the set of words 'visible from $v$ '. The central question of site percolation concerns the probability that $\mathcal{W}_{v} \ni 1^{\infty}$, where $1^{\infty}$ denotes the infinite word $111 \cdots$. The so-called AB-percolation problem concerns the existence in $\mathcal{W}_{v}$ of the infinite alternating word $01010 \cdots$, see [2].

More generally, for given $p$, we ask which words lie in the random set $\mathcal{W}_{v}$. Partial answers to this question may be found in three papers $[4,19,20]$ of Kesten and co-authors Benjamini, Sidoravicius, and Zhang, and their results are summarised here as follows.

For $\mathbb{Z}^{d}$, with $p=\frac{1}{2}$ and $d$ sufficiently large, we have from [4] that

$$
\mathbb{P}_{\frac{1}{2}}\left(\mathcal{W}_{0}=\mathcal{W}\right)>0
$$


and indeed there exists (a.s.) some vertex $v$ for which $\mathcal{W}_{v}=\mathcal{W}$. Partial results are obtained for $\mathbb{Z}^{d}$ with edge-orientations in increasing coordinate directions.

For the triangular lattice $\mathbb{T}$ and $p=\frac{1}{2}$, we have from [19] that

$$
\mathbb{P}_{\frac{1}{2}}\left(\bigcup_{v \in V} \mathcal{W}_{v} \text { contains almost every word }\right)=1,
$$

where the set of words seen includes all periodic words apart from $0^{\infty}$ and $1^{\infty}$. The measure on $\mathcal{W}$ can be taken in (1.8) as any non-trivial product measure. This extends the observation that AB-percolation takes place at $p=\frac{1}{2}$, whereas there is no infinite cluster in the usual site percolation model.

Finally, for the 'close-packed' lattice $\mathbb{Z}_{\mathrm{cp}}^{2}$ obtained from $\mathbb{Z}^{2}$ by adding both diagonals to each face,

$$
\mathbb{P}_{p}\left(\mathcal{W}_{0}=\mathcal{W}\right)>0
$$

for $1-p_{\mathrm{c}}<p<p_{\mathrm{c}}$, with $p_{\mathrm{c}}=p_{\mathrm{c}}\left(\mathbb{Z}^{2}\right)$. Moreover, every word is (a.s.) seen along some self-avoiding path in the lattice. See [20].

\section{Acknowledgements}

The author acknowledges the contributions of his co-authors Tom Liggett and Thomas Richthammer. He profited from discussions with Alexander Holroyd while at the Department of Mathematics at the University of British Columbia, and with Ron Peled and Vladas Sidoravicius while visiting the Institut Henri Poincaré-Centre Emile Borel, both during 2008. This article was written during a visit to the Section de Mathématiques at the University of Geneva, supported by the Swiss National Science Foundation. The author thanks Ron Peled for his comments on a draft.

\section{References}

[1] Andjel, E. 1992. Survival of multidimensional contact process in random environments. Bulletin of the Brazilian Mathematical Society, 23, 109119.

[2] Appel, M. J. B., and Wierman, J. C. 1993. AB percolation on bonddecorated graphs. Journal of Applied Probability, 30, 153-166.

[3] Balister, P. N., Bollobás, B., and Stacey, A. M. 2000. Dependent percolation in two dimensions. Probability Theory and Related Fields, 117, $495-513$. 
[4] Benjamini, I., and Kesten, H. 1995. Percolation of arbitrary words in $\{0,1\}^{\mathbb{N}}$. Annals of Probability, 23, 1024-1060.

[5] Benjamini, I., Pemantle, R., and Peres, Y. 1998. Unpredictable paths and percolation. Annals of Probability, 26, 1198-1211.

[6] Benjamini, I., Gurel-Gurevich, O., and Peled, R. 2008. On $k$ wise independent events and percolation. In preparation, see http://cims.nyu.edu/ peled/.

[7] Björnberg, J. E., and Grimmett, G. R. 2009. The phase transition of the quantum Ising model is sharp. Journal of Statistical Physics, 136, 231-273.

[8] Bramson, M., Durrett, R. T., and Schonmann, R. H. 1991. The contact process in a random environment. Annals of Probability, 19, 960-983.

[9] Broadbent, S. R., and Hammersley, J. M. 1957. Percolation processes I. Crystals and mazes. Proceedings of the Cambridge Philosophical Society, 53, 629-641.

[10] Coppersmith, D., Tetali, P., and Winkler, P. 1993. Collisions among random walks on a graph. SIAM Journal on Discrete Mathematics, 6, 363-374.

[11] Gács, P. 2000. The clairvoyant demon has a hard task. Combinatorics, Probability and Computing, 9, 421-424.

[12] Gács, P. 2004. Compatible sequences and a slow Winkler percolation. Combinatorics, Probability and Computing, 13, 815-856.

[13] Grimmett, G. R. 1999. Percolation. 2nd edn. Berlin: Springer.

[14] Grimmett, G. R. 2008. Probability on Graphs. http://www.statslab. cam.ac.uk/ grg/books/pgs.html.

[15] Grimmett, G. R., Liggett, T. M., and Richthammer, T. 2008. Percolation of arbitrary words in one dimension. Random Structures $\&$ Algorithms. Arxiv:0807.1676.

[16] Grimmett, G. R., Osborne, T. J., and Scudo, P. F. 2008. Entanglement in the quantum Ising model. Journal of Statistical Physics, 131, 305-339.

[17] Hoffman, C. 2005. Phase transition in dependent percolation. Communications in Mathematical Physics, 254, 1-22.

[18] Jonasson, J., Mossel, E., and Peres, Y. 2000. Percolation in a dependent random environment. Random Structures \& Algorithms, 16, 333-343.

[19] Kesten, H., Sidoravicius, V., and Zhang, Y. 1998. Almost all words are seen in critical site percolation on the triangular lattice. Electronic Journal of Probability, 3, 1-75. Paper \#10.

[20] Kesten, H., Sidoravicius, V., and Zhang, Y. 2001. Percolation of arbitrary words on the close-packed graph of $\mathbb{Z}^{2}$. Electronic Journal of Probability, 6, 1-27. Paper \#4.

[21] Kingman, J. F. C. 1968. The ergodic theory of subadditive stochastic processes. Journal of the Royal Statistical Society B, 30, 499-510.

[22] Kingman, J. F. C. 1973. Subadditive ergodic theory. Annals of Probability, 1, 883-909.

[23] Kingman, J. F. C. 1982. On the genealogy of large populations. Journal of Applied Probability, 19A, 27-43. 
[24] Kingman, J. F. C. 2000. Origins of the coalescent: 1974-1982. Genetics, 156, 1461-1463.

[25] Klein, A. 1994a. Extinction of contact and percolation processes in a random environment. Annals of Probability, 22, 1227-1251.

[26] Klein, A. 1994b. Multiscale analysis in disordered systems: percolation and contact process in random environment. Pages 139-152 of: Grimmett, G. R. (ed), Disorder in Physical Systems. Dordrecht: Kluwer.

[27] Liggett, T. M. 1985. Interacting Particle Systems. Berlin: Springer.

[28] Liggett, T. M. 1999. Stochastic Interacting Systems: Contact, Voter and Exclusion Processes. Berlin: Springer.

[29] Lima, B. N. B. de. 2008. A note about the truncation question in percolation of words. Bulletin of the Brazilian Mathematical Society, 39, 183-189.

[30] Lima, B. N. B. de, Sanchis, R., and Silva, R. W. C. 2009. Percolation of words on $\mathbb{Z}^{d}$ with long range connections. Arxiv:0905.4615.

[31] Newman, C. M., and Volchan, S. 1996. Persistent survival of onedimensional contact processes in random environments. Annals of Probability, 24, 411-421.

[32] Peled, R. 2008. Personal communication.

[33] Peled, R. 2009. On rough isometries of Poisson processes on the line. Annals of Applied Probability. Arxiv:0709.2382.

[34] Spitzer, F. L. 1976. Principles of Random Walk. 2nd edn. New York: Springer.

[35] Werner, W. 2007. Lectures on two-dimensional critical percolation. Arxiv:0710.0856.

[36] Winkler, P. 2000. Dependent percolation and colliding random walks. Random Structures \& Algorithms, 16, 58-84. 